\title{
The Fourier Transform and Principles of Quantum Mechanics
}

\author{
Do Tan Si \\ Ho Chi Minhcity Physical Association, Ho Chi Minhcity, Vietnam \\ Email: tansi_do@yahoo.com
}

How to cite this paper: Si, D.T. (2018) The Fourier Transform and Principles of Quantum Mechanics. Applied Mathematics, 9, 347-354.

https://doi.org/10.4236/am.2018.94026

Received: March 12, 2018

Accepted: April 15, 2018

Published: April 18, 2018

Copyright (C) 2018 by author and Scientific Research Publishing Inc. This work is licensed under the Creative Commons Attribution International License (CC BY 4.0).

http://creativecommons.org/licenses/by/4.0/

\begin{abstract}
From the hypotheses that the position-representation of a physical state is the Fourier transform of its momentum-representation and that the timerepresentation is the inverse Fourier transform of its energy-representation, we are able to obtain the Planck relation $E=h v$, the de Broglie relation $p=h / \lambda$, the Dirac fundamental commutation relation, the Schrödinger equations, the Heisenberg uncertainty principle in quantum mechanics, and the annihilation/creation of a photon from excitation/de-excitation of an atom following Bohr.
\end{abstract}

\section{Keywords}

Fourier Transform of Bra, Obtaining Dirac Commutation Relation and Principles of Quantum Mechanics

\section{Introduction}

In Quantum Mechanics [1], it is postulated that a physical state is represented by a state vector containing all the information about it. This complex vector is denominated ket following Dirac and is denoted by the symbol $|\alpha\rangle$. To the kets space is associated a dual space containing vectors called bras and denoted by $\langle\alpha|$ in the similar manner as in Hermitian conjugation a row vector is formed with the complex conjugates of elements of a column vector. An inner product between a bra and a ket is then defined and denoted by the bracket $\langle\beta \mid \alpha\rangle$, similar to the scalar product of two functions $\int_{-\infty}^{+\infty} f^{*}(x) g(x) \mathrm{d} x$.

An observable, such as momentum components, or more precisely the measure of an observable, may be represented by an operator which acts on a ket from the left or on a bra from the right. 
The principle of measurement stipulates that if in a state $|\alpha\rangle$ an observable $A$ has a definite value then applying the operator $A$ on $|\alpha\rangle$ will let the state intact, i.e. $|\alpha\rangle$ is an eigenket of $A$ corresponding to the eigenvalue a

$$
A|\alpha\rangle=a|\alpha\rangle
$$

For simplicity we consider particles moving in a one-dimensional space and will generalize for three-dimensional space whenever it is necessary.

Let $\hat{X}, \hat{P}_{x}, \hat{T}, \hat{H}$ be respectively the operators representing the measurements of the $X$-component of the position $\boldsymbol{r}$, the x-component of the momentum $\boldsymbol{p}$, the lap of time $t$ counted from some origine of time and the energy $E$ of a particle. The set of eigenkets of these operators are denoted by $|x\rangle,|p\rangle,|t\rangle,|E\rangle$ where we write $|p\rangle$ instead of $\left|p_{x}\right\rangle$ for simplicity whenever we work in one dimensional (1D) space

$$
\begin{aligned}
\hat{X}|x\rangle & =x|x\rangle, \quad \hat{P}_{x}|p\rangle=p|p\rangle \\
\hat{T}|t\rangle & =t|t\rangle, \hat{H}|E\rangle=E|E\rangle
\end{aligned}
$$

The eigenvalues of these operators are real numbers so that the state space is nondenumerably indefinite, called Hilbert space.

The inner product $\langle x \mid \alpha\rangle$ for example is a function of $x$, depending on the parameters containing in $|\alpha\rangle$ and is called position-representation or wave function of $|\alpha\rangle$. Similarly $\langle p \mid \alpha\rangle$ is called momentum-representation of $|\alpha\rangle$.

The quantities $|\langle x \mid \alpha\rangle|^{2} \mathrm{~d} x,|\langle t \mid \alpha\rangle|^{2} \mathrm{~d} t,|\langle p \mid \alpha\rangle|^{2} \mathrm{~d} p,|\langle E \mid \alpha\rangle|^{2} \mathrm{~d} E$ represent respectively the probability for finding the particle in a state $|\alpha\rangle$ inside the interval $(x-\mathrm{d} x / 2, x+\mathrm{d} x / 2)$, inside the interval of time $(t-\mathrm{d} t / 2, t+\mathrm{d} t / 2)$, the value of its momentum between $p-\mathrm{d} p / 2$ and $p+\mathrm{d} p / 2$ and of its energy between $E-\mathrm{d} E / 2$ and $E+\mathrm{d} E / 2$.

Lastly, it is important to remark that each set of eigenkets $\{|x\rangle\},\{|p\rangle\},\{|t\rangle\},\{|E\rangle\}$ constitutes a basis for spanning the whole Hilbert space of states, so that a ket may be represented by its components on each of these bases, for examples

$$
\begin{array}{r}
\langle x \mid \alpha\rangle=\int_{-\infty}^{+\infty} \mathrm{d} x|x\rangle\langle x \mid \alpha\rangle \\
\langle t \mid \alpha\rangle=\int_{-\infty}^{+\infty} \mathrm{d} t|t\rangle\langle t \mid \alpha\rangle
\end{array}
$$

The aim of this work is to study the relationships between different modes of representation of a state. We obtain then from the hypothesis saying that $\langle x \mid \alpha\rangle$ is the Fourier transform [2] of $\langle p \mid \alpha\rangle$ and $\langle t \mid \alpha\rangle$ the inverse Fourier transform of $\langle E \mid \alpha\rangle$ the principles of quantum mechanics [1] [2] [3] which are the Planck relation $E=h v$, the de Broglie relation $p=\frac{h}{\lambda}$, the Dirac fundamental commutation relation $\left[\hat{P}_{x}, \hat{X}\right]=-i \hbar \hat{I}$, the Shrödinger equations, the creation of a photon from deexcitation of an atom following Bohr, the Heisenberg uncertainty principle. 


\section{Fourier Relationships between Different Representations}

Let us consider the state $|\alpha\rangle$ of a universe fulfilled with identical particles having the same momentum $\boldsymbol{p}_{0}$. This state corresponds to a wave function $\langle\boldsymbol{p} \mid \alpha\rangle$ which is equal to zero anywhere except at the point $\boldsymbol{p}_{0}$ in the momentumspace, i.e. a Dirac delta function. As the particle can take any place in space its position-representation $\langle\boldsymbol{r} \mid \alpha\rangle$ is equal to unity at any position in space. The relationship between $\langle\boldsymbol{r} \mid \alpha\rangle$ and $\langle\boldsymbol{p} \mid \alpha\rangle$ is then somehow a Fourier transformation [4] [5] which, as we know, transforms the function $(2 \pi)^{\frac{1}{2}} \delta(p)$ into the function $u(x)=1$.

Generalizing for all other states, we propose the following primordial hypotheses.

\section{Hypotheses}

"The position-representation of a physical state is the Fourier transform, denoted by FT, of its momentum-representation"

$$
\langle\boldsymbol{r} \mid \alpha\rangle=(2 \pi)^{-\frac{3}{2}} \int_{\mathfrak{R}^{3}} \mathrm{e}^{i \theta \boldsymbol{p r}}\langle\boldsymbol{p} \mid \alpha\rangle \mathrm{d} \theta \boldsymbol{p}=F T\langle\boldsymbol{p} \mid \alpha\rangle
$$

In Equation (2.1) we have introduced a coefficient $\theta$ to assure that the scalar product $\theta \boldsymbol{p r}$ is dimensionless.

By the same approach, the case where a particle may conserve one constant value $E_{0}$ for its energy although time flows from $-\infty$ to $+\infty$ leads us to the hypothesis that $\langle t \mid \alpha\rangle$ is the inverse Fourier transform of $\langle E \mid \alpha\rangle$.

$$
\langle t \mid \alpha\rangle=(2 \pi)^{-\frac{1}{2}} \int_{-\infty}^{+\infty} \mathrm{e}^{-i \theta E t}\langle E \mid \alpha\rangle \mathrm{d} \theta E=F T^{-1}\langle E \mid \alpha\rangle
$$

In Equation (2.2) the use of the inverse Fourier transform instead of the Fourier transform itself is for conformity with the familiar form of a planar wave $\exp i 2 \pi\left(\lambda^{-1} x-v t\right)$.

Because $|\alpha\rangle$ is arbitrary the hypotheses (2.1), (2.2) may smartly be written

$$
\begin{aligned}
& \langle\boldsymbol{r}|=F T\langle\boldsymbol{p}| \\
& \langle t|=F T^{-1}\langle E|
\end{aligned}
$$

We notify that the Fourier relationship (2.1) may be deduced from the accepted laws of commutation $[\hat{P}, \hat{X}]=-i \hbar \hat{I}$ established by Dirac in $1925[1]$ by analogy with the Poisson brackets in classical mechanics. On the contrary, in this work we consider Equations (2.1), (2.2) as the hypotheses from which one may deduce the Dirac fundamental commutation relations among other applications as presented in the next chapter where the main principles of Quantum Mechanics are derived.

\section{Applications}

\subsection{Obtaining the Planck and De Broglie Principles}

From the hypothesis (2.1), (2.2) we obtain the time-representation of the state of 
a particle having a constant energy $E_{0}$ by taking the inverse Fourier transform of

$$
\begin{aligned}
& \left\langle E \mid E_{0}\right\rangle=\delta\left(E-E_{0}\right) \\
& \left\langle t \mid E_{0}\right\rangle=(2 \pi)^{-\frac{1}{2}} \mathrm{e}^{-i \theta E_{0} t}
\end{aligned}
$$

and, similarly for a particle having a constant momentum in space:

$$
\left\langle\boldsymbol{r} \mid \boldsymbol{p}_{0}\right\rangle=(2 \pi)^{-\frac{3}{2}} \mathrm{e}^{i \theta \boldsymbol{p}_{0} \boldsymbol{r}}
$$

These equations show that a state with constant energy $E$ with respect to time corresponds to an oscillation in time with a period $T$ verifying

$$
\frac{2 \pi}{T}=2 \pi \nu=\theta E
$$

and a state with constant momentum $\boldsymbol{p}$ in 3D space corresponds to an oscillation in space with a wavelength $\lambda$ verifying

$$
\frac{2 \pi}{\lambda}=\theta p
$$

With the choice $\theta=\hbar^{-1}$, these conclusions are precisely the principles of Planck $E=h v$ and of de Broglie $p=h / \lambda$ [1] [2].

It is curious to observe that to the ket $|x\rangle$ one may assign the dimension $L^{\frac{1}{2}}$ and to the bra $\langle x|$ the dimension $L^{-\frac{1}{2}}$. Similarly, the dimensions of $|p\rangle,|t\rangle,|E\rangle$ are respectively the square root of those of $\mathrm{p}, \mathrm{t}, \mathrm{E}$.

Attempting to understand more about the particle-wave relationship, we may imagine that in order to maintain E constant in time and $\boldsymbol{p}$ constant in space, a particle has to appear and disappear periodically between the real world and an imaginary world. This periodic movement creates a wave with frequency $v$ proportional to $\mathrm{E}$ and wavelength $\lambda$ proportional to $p^{-1}$. Inversely, a planar wave is the proof of existence of a particle with well defined $E$ and p. For light which has a certain colour, the corresponding particle is the photon.

\subsection{Obtaining the Dirac Fundamental Commutation Relations}

Beside $\hat{X}$ and $\hat{P}$ let us formally define another operator $\hat{D}_{x}$ by the relation

$$
\hat{D}_{x} \hat{X}-\hat{X} \hat{D}_{x} \equiv \hat{I}
$$

where $\hat{I}$ is the identity operator in the Hilbert space of state kets.

Now, in the 1D space of wave functions let $\breve{X}$ be the operator of multiplication by $x$ well defined in [6] and $\breve{D}_{x}$ the derivative operator

$$
\begin{gathered}
\breve{X} f(x)=x f(x), \breve{D}_{x} f(x)=f^{\prime}(x) \\
{\left[\breve{D_{x}}, \breve{X}\right] \equiv \breve{D}_{x} \breve{X}-\breve{X} \breve{D}_{x} \equiv \breve{I}}
\end{gathered}
$$

In the same way, in the $1 \mathrm{D}$ space of functions in momentum $f(p)$ we may define $\breve{P}$ as the operator of multiplication by $p$ and $\breve{D}_{p}$ as the operator of de- 
rivative with respect to $p$.

We must be attentive on the fact that the operators $\breve{X}, \breve{D}_{x}, \breve{P}, \breve{D}_{p}$ act on functions and $\hat{X}, \hat{D}_{x}, \hat{P}, \hat{D}_{p}$ act on bras and kets.

Now, from Equation (3.3) we get

$$
\left\langle x\left|\hat{D}_{x} \hat{X}-\hat{X} \hat{D}_{x}\right| x_{0}\right\rangle=\left(x_{0}-x\right)\left\langle x\left|\hat{D}_{x}\right| x_{0}\right\rangle=\left\langle x|\hat{I}| x_{0}\right\rangle=\delta\left(x-x_{0}\right)
$$

On the other hand, because $\breve{X} \delta(x)=x \delta(x)=0$ we have

$$
\begin{gathered}
\left(\breve{X}-x_{0} \breve{I}\right) \delta\left(x-x_{0}\right)=\left(x-x_{0}\right) \delta\left(x-x_{0}\right) \\
\breve{X} \delta\left(x-x_{0}\right)=x \delta\left(x-x_{0}\right)=x_{0} \delta\left(x-x_{0}\right)
\end{gathered}
$$

and, by applying Equation (3.7) onto the delta function $\delta\left(x-x_{0}\right)$ we get

$$
\left(x_{0}-x\right) \breve{D}_{x} \delta\left(x-x_{0}\right)=\delta\left(x-x_{0}\right)
$$

Replacing $\delta\left(x-x_{0}\right)$ by $\left\langle x \mid x_{0}\right\rangle$ in (3.9), (3.10) and utilizing (3.8) we get

$$
\begin{gathered}
\breve{X}\left\langle x \mid x_{0}\right\rangle=x_{0}\left\langle x \mid x_{0}\right\rangle=\left\langle x|\hat{X}| x_{0}\right\rangle \\
\left(x_{0}-x\right) \breve{D}_{x}\left\langle x \mid x_{0}\right\rangle=\left(x_{0}-x\right)\left\langle x\left|\hat{D}_{x}\right| x_{0}\right\rangle
\end{gathered}
$$

i.e., as $x_{0}$ is arbitrary

$$
\begin{aligned}
& \langle x| \hat{X} \equiv \breve{X}\langle x| \\
& \langle x| \hat{D}_{x} \equiv \breve{D}_{x}\langle x|
\end{aligned}
$$

The relations (2.3), (2.4), (3.11), (3.12) and the following properties of the Fourier transform one may find in [5] where the FT form

$$
\operatorname{FTf}(x)=\frac{1}{\sqrt{2 \pi}} \int_{-\infty}^{+\infty} \mathrm{e}^{-i k x} f(k) \mathrm{d} k
$$

is represented by the operator

$$
F T \equiv \mathrm{e}^{\frac{\mathrm{i} \pi}{4}} \mathrm{e}^{-\frac{i}{2} \breve{X}^{2}} \mathrm{e}^{\frac{i}{2} \breve{D}_{x}^{2}} \mathrm{e}^{-\frac{i}{2} \breve{X}^{2}}
$$

which, utilizing the fundamental identity in Operator calculus [7]

$$
f\left(\breve{D}_{x}\right) g(\breve{X}) \equiv g(\breve{X}) f\left(\breve{D}_{x}\right)+\sum_{k=1}^{\infty} \frac{1}{k !} g^{(k)}(\breve{X}) f^{(k)}\left(\breve{D}_{x}\right)
$$

transforms $\breve{X}$ into

$$
\begin{aligned}
& F T \breve{X} F T^{-1} \equiv \mathrm{e}^{\frac{i \pi}{4}} \mathrm{e}^{-\frac{i}{2} \breve{X}^{2}} \mathrm{e}^{\frac{i}{2} \breve{D}_{x}^{2}} \mathrm{e}^{-\frac{i}{2} \breve{X}^{2}} \breve{X} \mathrm{e}^{+\frac{i}{2} \breve{X}^{2}} \mathrm{e}^{-\frac{i}{2} \breve{D}_{x}^{2}} \mathrm{e}^{+\frac{i}{2} \breve{X}^{2}} \mathrm{e}^{-\frac{i \pi}{4}} \\
& \equiv \mathrm{e}^{-\frac{i}{2} \breve{X}^{2}} \mathrm{e}^{\frac{i}{2} \breve{D}_{x}{ }^{2}} \breve{X} \mathrm{e}^{-\frac{i}{2} \breve{D}_{x}{ }^{2}} \mathrm{e}^{+\frac{i}{2} \breve{X}^{2}} \equiv \mathrm{e}^{-\frac{i}{2} \breve{X}^{2}}\left(\breve{X}+i \breve{D}_{x}\right) \mathrm{e}^{+\frac{i}{2} \breve{X}^{2}} \equiv i \breve{D}_{x}
\end{aligned}
$$

In the context of the present work we change $\breve{X}$ into $\breve{X} / \hbar$ and $\breve{D}_{x}$ into $\hbar \breve{D}_{x}$ so that

$$
\begin{gathered}
F(p)=F T f(x)=\frac{1}{\hbar \sqrt{2 \pi}} \int_{-\infty}^{+\infty} \mathrm{e}^{i p x / \hbar} f(x) \mathrm{d} x \\
F T x g(x)=F T \breve{X} F T^{-1} F \operatorname{FT}(x)=-i \hbar \breve{D}_{p} F T g(x) \\
F T f(x) g(x)=f\left(-i \hbar \breve{D}_{p}\right) F T g(x)
\end{gathered}
$$


Equations (3.18), (3.12) lead to

$$
\begin{aligned}
\left\langle x|\hat{P}| p_{0}\right\rangle & =F T\left\langle p|\hat{P}| p_{0}\right\rangle=F T p\left\langle p \mid p_{0}\right\rangle=-i \hbar \breve{D}_{x} F T\left\langle p \mid p_{0}\right\rangle \\
& =-i \hbar \breve{D}_{x}\left\langle x \mid p_{0}\right\rangle=\left\langle x\left|-i \hbar \hat{D}_{x}\right| p_{0}\right\rangle
\end{aligned}
$$

Similarly, by repeating the reasoning with $\hat{X}, \hat{D}_{p}$ we get

$$
\begin{aligned}
\left\langle p|\hat{X}| p_{0}\right\rangle & =F T^{-1} x\left\langle x \mid p_{0}\right\rangle=i \hbar \breve{D}_{p} F T^{-1}\left\langle x \mid p_{0}\right\rangle \\
& =i \hbar \breve{D}_{p}\left\langle p \mid p_{0}\right\rangle=\left\langle p\left|i \hbar \hat{D}_{p}\right| p_{0}\right\rangle
\end{aligned}
$$

The above relations hold for any value of $\left|p_{0}\right\rangle$ so that by (3.19), (3.20) we may state: "In the Hilbert kets space of physical states

$$
\hat{P} \equiv-i \hbar \nabla_{r} ; \hat{r} \equiv i \hbar \nabla_{p} ; \hat{H} \equiv i \hbar \partial_{t} ; \hat{T} \equiv-i \hbar \partial_{E} " .
$$

These equivalence relations in turn lead to the Dirac fundamental commutation relations in quantum mechanics

$$
\left[\hat{P}_{i}, \hat{r}_{j}\right]=-i \hbar \delta_{i j} \hat{I},[\hat{H}, \hat{T}]=i \hbar \hat{I}
$$

\subsection{Obtaining the Schrödinger Equations}

From the relations (3.11), (3.12) we may also get an important proposition

"The eigenvalue equation

$$
A(\hat{X}, \hat{P})|\alpha\rangle=a|\alpha\rangle
$$

of an arbitrary operator $A(\hat{X}, \hat{P})$ leads to the differential equation (or partial differential equation in the cases of $3 \mathrm{D}$ space) for the corresponding wave function

$$
\langle x|A(\hat{X}, \hat{P})| \alpha\rangle=A\left(\breve{X},-i \hbar \breve{D}_{x}\right)\langle x \mid \alpha\rangle=a\langle x \mid \alpha\rangle "
$$

For example, with $A(\hat{X}, \hat{P}) \equiv \frac{1}{2 m} \hat{P}^{2}+V(\hat{X})$, we obtain the Schrödinger equation

$$
\left(-\frac{\hbar^{2}}{2 m} \breve{D}_{x}^{2}+V(x)\right) \Psi(x)=E \Psi(x)
$$

As for the time dependent Schrödinger equation, it may be written down from Equations (3.11), (3.12)

$$
i \hbar \partial_{t}\langle x, t \mid \Psi\rangle=\left(-\frac{\hbar^{2}}{2 m} \frac{\partial^{2}}{\partial x^{2}}+V(x, t)\right)\langle x, t \mid \Psi\rangle
$$

\subsection{Obtaining the Heisenberg Uncertainty Principles}

A state $|\alpha\rangle$ where $\left(p_{0}-\Delta p / 2\right) \leq p \leq\left(p_{0}+\Delta p / 2\right)$ corresponds to the convolution product

$$
\langle p \mid \alpha\rangle=\delta\left(p-p_{0}\right) \otimes S q(p, \Delta p)
$$

where $S q(p, d)$ is the square function, equal to zero for $|p|>d / 2$ and to $d^{-1}$ 
for $|p| \leq d / 2$.

By Fourier transform we get

$$
\langle x \mid \alpha\rangle=(2 \pi)^{-1} \mathrm{e}^{i \frac{p_{0} x}{\hbar}} \frac{\sin (x \Delta p / 2 \hbar)}{x \Delta p / 2 \hbar}
$$

The function in the right member has absolute value equal to $(2 \pi)^{-1}$ for $x=0$, vanishes for $x \Delta p= \pm 2 \hbar \pi$, so that its noticeable values are situated in the interval

$$
\Delta x \approx 2 \hbar \pi / \Delta p=h / \Delta p
$$

The Heisenberg uncertainty principle

$$
\Delta x \Delta p \geq \hbar
$$

is deduced from the last equation.

Similarly for the proof of

$$
\Delta E \Delta t \geq \hbar
$$

\subsection{Obtaining the Emission of Photons by Atoms Following Bohr}

Consider a state $|\alpha\rangle$ that has many stable values for its energy and suppose that $|\alpha\rangle$ is the sum of individual states each of them having only one value of energy

$$
\langle E \mid \alpha\rangle=\sum_{j=1}^{N} \delta\left(E-E_{j}\right)
$$

By Fourier transform we get

$$
\langle t \mid \alpha\rangle=(2 \pi)^{-\frac{1}{2}} \sum_{j=1}^{N} \mathrm{e}^{-i E_{j} t / \hbar}
$$

so that

$$
|\langle t \mid \alpha\rangle|^{2}=(2 \pi)^{-1}\left(N+\sum_{j=1}^{N} \sum_{k=1}^{j-1} 2 \cos \left(E_{k}-E_{j}\right) t / \hbar\right) .
$$

By Equation (3.31) we see that the probability for observing $|\alpha\rangle$ at the instant $t$ has multiperiodc peeks at

$$
t= \pm n \frac{2 \pi \hbar}{E_{k}-E_{j}}, \forall j<k=1,2, \cdots, N ; n=0,1,2, \cdots
$$

According to Equation (3.32) one may observe in addition to the wave $v_{i}=h^{-1} E_{i}$ other waves with frequencies $v_{j k}=h^{-1}\left(E_{k}-E_{j}\right)$. Thus, the realization of the change in energy may give rise to the emission or absorption of a photon $h v_{j k}$, conforming to the hypothesis of N. Bohr.

\section{Conclusions}

We think that the hypotheses of complex state vectors, the principles of measurement of physical observables and of mixing of states, the use of Hilbert kets space and the hypotheses on position-momentum and energy-time Fourier rela- 
tionships discussed in this work may considerably simplify the comprehension of the principles of Quantum Mechanics conceived by Planck, de Broglie, Dirac, Heisenberg, Bohr.

Remarkably, we see also that the use of the property of the Fourier transform say $\operatorname{FTx} f(x)=-i \hbar D_{p} F T f(x)$ leads to the Dirac fundamental commutation relations and the Schrödinger equations.

Furthermore, linked to this work we may cite the unification of many laws in wave optics in only one theorem involving principally the Fourier transform in 3D space [8].

We wish that more and more readers would consider the Fourier transform not only as an integral transform [9] but also as a differential transform, which has many interesting properties [5].

\section{Acknowledgements}

The author is grateful toward regretted Prof. Marcel Demeur ${ }^{\dagger}$, Service Physique Théorique Nucléaire, Univ. Libre de Bruxelles for formation and helps in the past.

\section{References}

[1] Dirac, P.A.M. (1960) Quantum Mechanics. 4th Edition, Oxford University Press, Oxford.

[2] Shankar, R. (1994) Principles of Quantum Mechanics. Springer. https://doi.org/10.1007/978-1-4757-0576-8

[3] Griffiths, D.J. (1995) Introduction to Quantum Mechanics. Prentice Hall Inc., Upper Saddle River.

[4] Bracewell, R. (1999) The Fourier Transform and Its Applications. 3rd Edition, McGraw-Hill, New York.

[5] Do, T.S. (2015) Differential Transforms. The Case of the Fourier Transform. Applied Physics Research, 7, 137-153. https://doi.org/10.5539/apr.v7n2p137

[6] Eckart, C. (1926) Operator Calculus and the Solutions of the Equations of Quantum Dynamics. Physical Review, 28, 711-726. https://doi.org/10.1103/PhysRev.28.711

[7] Do, T.S. (2016) Differential Calculus for Differential Equations, Special Functions, Laplace Transform. Applied Physics Research, 8, No. 1. https://doi.org/10.5539/apr.v8n1p158

[8] Do, T.S. (2014) On Amplitude of Fraunhofer Diffractions of Waves by 3D Objects. Journal of Optics, 43, 219-230.

[9] Wolf, K.B. (1979) Integral Transforms in Science and Engineering. Plenum Press, New York. https://doi.org/10.1007/978-1-4757-0872-1 